\title{
Komogorov-Smirnov Test
}

National Cancer Institute

\section{Source}

National Cancer Institute. Komogorov-Smirnov Test. NCI Thesaurus. Code C53250.

Either the Kolmogorov-Smirnov one-sample test, a goodness-of-fit test, that tests

whether an observed dataset is consistent with an hypothesized theoretical distribution

or the Kolmogorov-Smirnov two-sample test, a test of the null hypothesis that two

independent samples have been drawn from the same population (or from populations with the same distribution). 